\title{
A Summary of the Lateral Cutoff Analysis and Results from NASA's Farfield Investigation of No-boom Thresholds
}

\author{
Larry J. Cliatt, II, ${ }^{1, \mathrm{a}}$ Michael A. Hill, ${ }^{1, \mathrm{~b}}$ Edward A. Haering, Jr., ${ }^{1, \mathrm{c}}$ Sarah R. Arnac ${ }^{1, \mathrm{~d}}$ \\ ${ }^{I}$ NASA Armstrong Flight Research Center, Edwards, California, USA 93523 \\ a larry.j.cliatt@nasa.gov \\ bmichael.a.hill-1@nasa.gov \\ cedward.a.haering@nasa.gov \\ dsarah.arnac@nasa.gov
}

\begin{abstract}
In support of the ongoing effort by the National Aeronautics and Space Administration (NASA) to bring supersonic commercial travel to the public, NASA, in partnership with other industry organizations, conducted a flight research experiment to analyze acoustic propagation at the lateral edge of the sonic boom carpet. The name of the effort was the Farfield Investigation of No-boom Thresholds (FaINT). The research from FaINT determined an appropriate metric for sonic boom waveforms in the transition and shadow zones called Perceived Sound Exposure Level, established a value of $65 \mathrm{~dB}$ as a limit for the acoustic lateral extent of a sonic boom's noise region, analyzed change in sonic boom levels near lateral cutoff, and compared between real sonic boom measurements and numerical predictions.
\end{abstract}

\section{BACKGROUND AND TEST OBJECTIVES}

The Farfield Investigation of No-boom Thresholds (FaINT) effort aligned with the need of the aerospace industry to continue to understand the entire envelope of sonic boom propagation. The primary sonic boom carpet is only one component of acoustic disturbances classified as sonic booms. Sonic booms in the shadow side have been challenging to analyze due to their complex propagation. ${ }^{1}$ The FaINT test attempted to analyze the shadow side propagations, shown in Fig. 1, where typical N-wave sonic boom pressure signatures transition into evanescent waves (sound waves that exponentially attenuate) laterally beyond the bounds of the primary sonic boom carpet.

The FaINT test was a National Aeronautics and

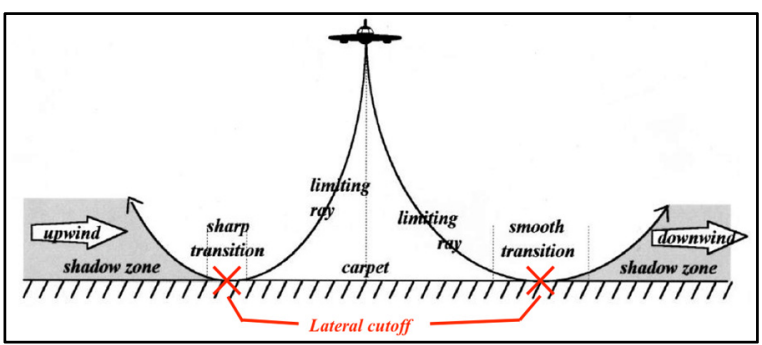

FIGURE 1. Lateral cutoff and transition region. ${ }^{1}$ Space Administration (NASA) collaborative effort with several industry partners. Partners included Wyle (El Segundo, California, USA); The Boeing Company (Chicago, Illinois, USA); Gulfstream Aerospace Corporation (Savannah, Georgia, USA); The Cessna Aircraft Company (Wichita, Kansas, USA); The Pennsylvania State University (University Park, Pennsylvania, USA); The Japan Aerospace Exploration Agency (Chōfu, Tokyo, Japan) and Dassault Aviation (Paris, France).

Examining acoustic propagation at the lateral edge of the sonic boom carpet will help the aerospace industry realize the full extent and ranges of noises generated by supersonic airplanes. Considerable sound levels can be experienced far beyond the sonic boom carpet predicted by computer models. Understanding the entire physical area affected by supersonic airplanes will be critical in determining target flight profiles and airplane designs for future commercial supersonic airplanes. This paper focuses on quantifying the lateral extent of noise beyond a sonic boom's carpet by defining an "acoustic lateral cutoff" threshold using newly identified appropriate metrics for transition and shadow zone pressure signatures, as well as comparisons between real sonic boom measurements and numerical predications. 


\section{TEST ARCHITECTURE}

The primary ground sensors for FaINT consisted of a linear microphone array with 60 microphones spaced $125 \mathrm{ft}(38 \mathrm{~m})$ apart, perpendicular to the airplane flight path. Brüel \& Kjærr (B\&K) (Naerum, Denmark) model 4193 low-frequency microphones (without the UC0211 low-frequency adapter) were used on the microphone array at 47 locations, and GRAS model 40AN low-frequency microphones were used at 13 locations.

A standard F-18B airplane configured with a centerline fuel tank was used to generate the sonic booms, and realtime airplane time-speed-position information data were recorded. To capture the lateral cutoff region of the sonic boom carpet, the F-18B airplane was flown supersonically, straight and level, as to generate a consistent shock that would intersect the microphone array. For each pass, it was attempted to place the lateral cutoff region along the ground array so as to capture the full $\mathrm{N}$-wave shock along with transition region and shadow zone.

For mission planning, PCBoom, ${ }^{2}$ a sonic boom propagation prediction computer package, was used to give a theoretical sonic boom footprint. The predicted F-18B airplane waypoint was translated to place the lateral edge of the sonic boom on the microphone array. Lateral cutoff tests for FaINT consisted of seven flights. There were 37 lateral cutoff flight passes total, ranging from 35,300-40,600 ft (10,800-12,400 m) Hp and Mach 1.223-1.286.

\section{ANALYSIS AND RESULTS}

Lateral cutoff occurs at the sonic boom carpet edge when the rays no longer reach the ground. ${ }^{3}$ In raytracing terms used by some computer models, sonic booms propagate at different azimuths (phi angles) from the airplane. ${ }^{2}$

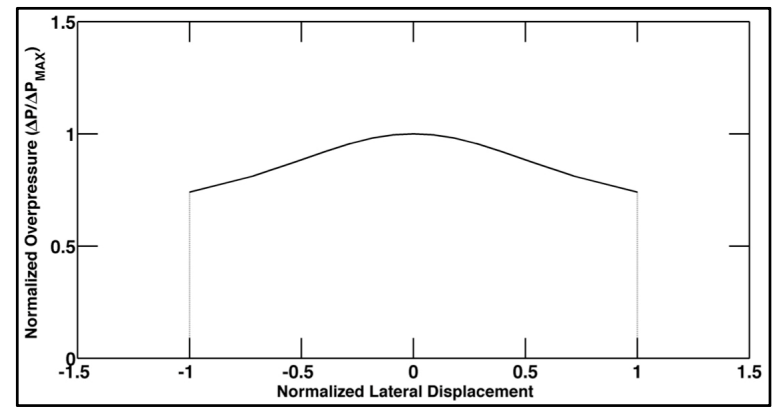

FIGURE 2. Nominal lateral sonic boom profile. 0 -deg phi is directly below the airplane and +/- 90-deg phi is projected from either wing. As the magnitude of phi increases, eventually rays no longer reach the ground. Figure 2 shows the predicted lateral sonic boom profile for an F-18B airplane at Mach 1.255, 36,750 ft $(11,201$ m) Hp, the median FaINT flight condition. The prediction was computed using PCBoom and U.S. Standard Atmospheric ${ }^{4}$ conditions. It should be noted that the pressure levels do not gradually decrease to zero; instead they abruptly end when the rays no longer reach the ground. FaINT intended to investigate what actually occurs at and beyond this lateral cutoff.

\section{Appropriate Metrics for Lateral Cutoff Acoustics}

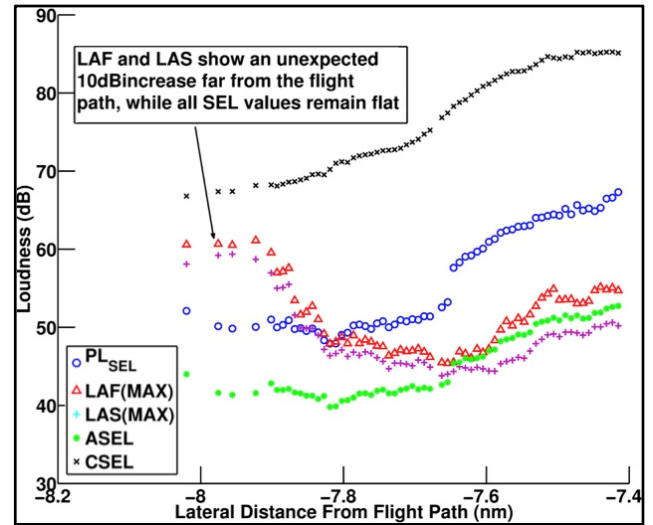

FIGURE 3. Comparison of acoustic metrics.
Metrics used by the sonic boom community, such as the $70 \mathrm{~ms}$ integrated ${ }^{5}$ Stevens' Mark VII ${ }^{6}$ perceived loudness $\left(\mathrm{PL}_{70}\right)$, may be less applicable for the waveform shape of the evanescent waves in the transition and shadow zones. The acoustic signature in these regions is highly variable in duration and impulsiveness and the short integration time of the $\mathrm{PL}_{70}$ metric is not well suited for these longer, duration-varying sounds.

An alternate acoustic metric may be more applicable for signatures of the type experienced in the lateral cutoff region. The metric uses Stevens' Perceived Level as a method of frequency weighting, with the input determined by Sound Exposure Level (SEL) $1 \mathrm{~s}$ normalized method of integration. ${ }^{7}$ This metric is referred to as Perceived Sound Exposure Level (PLSEL). Figure 3 shows a comparison between PLSEL and other commonly used sonic boom acoustic metrics near the sonic boom carpet edge.

These metrics were calculated using standard procedures described in ISO 1996-2 ${ }^{7}$ and ANSI S1.4. ${ }^{8}$ The plot shows acoustic metrics as a function of distance from the F-18B airplane flight path. In plots like these, lateral distance values are negative to correlate with the PCBoom ray tracing phi angle convention (i.e., negative values on the right side of the airplane). As shown, the PLSEL and CSEL values aligned with the expected acoustic profile beyond lateral 
cutoff better than the other metrics. Because of the better performance of the SEL metrics, along with Stevens' PL being an industry-accepted weighting for sonic boom measurements, PL $\mathrm{SEL}_{\mathrm{S}}$ was used to analyze sonic booms near lateral cutoff in this paper.

\section{Acoustic Lateral Cutoff and Lateral Cutoff Profile}

The concept of "acoustical lateral cutoff" is defined as the location to the side of the airplane track where the noise is no longer notable relative to ambient noise. To determine a quantitative acoustic lateral cutoff based on FaINT data, first an acoustic noise floor was determined. Data showed that, while the overpressures continue to decrease with

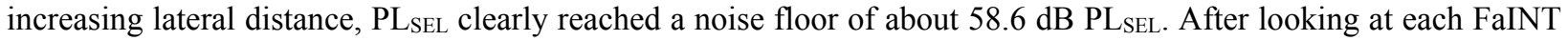
test point, it is apparent that at four times the acoustic power (or $+6 \mathrm{~dB}$ ) of the ambient noise, lateral sonic boom profile characteristics are consistently discernable. This observation led to the postulation that $65 \mathrm{~dB} \mathrm{PL}_{\mathrm{SEL}}$ is a reasonable threshold for defining acoustic lateral cutoff.

Real data knowledge of the sonic boom propagation at the lateral cutoff will give researchers an understanding of noise levels beyond where current, common sonic boom prediction tools are able to model. Airplanes with outer mold lines designed to produce "low sonic booms" (sonic booms intended to be comparable to future commercial supersonic airplanes) may produce unorthodox sonic boom carpets, with the peak levels several miles away from the airplane's flight path. If this is the case, knowledge of sonic boom propagation near the carpet edge is essential.

A majority of FaINT measurements demonstrated the expected evanescent decay profile of sonic boom magnitude with increasing distance from the airplane's flight path near lateral cutoff. Figure 4a illustrates a select example. The figure shows a steady, exponential-like decrease of both overpressure and PL $L_{S E L}$. Several important observations are derived from similar test points. First, for this case, the acoustic floor is at about $-4.7 \mathrm{~nm}$, when PL $\mathrm{SEL}_{\text {reaches }}$ the asymptote of ambient noise at about $60 \mathrm{PL}_{\mathrm{SEL}}$. Second, the data shows that the mission planning techniques are capable of accurately capturing the carpet edge. Third, the data supports the previously suggested $65 \mathrm{~dB}$ PL $\mathrm{LEL}_{\text {as }}$ a reasonable threshold for defining acoustic lateral cutoff. The well-behaved nature of the profile in Fig. 4a is likely due to the lack of strong temperature inversion near the ground. Several other possible influences were investigated, including atmospheric turbulence, steadiness of flight, and wind speed and direction at ground level.

The weakness of a temperature inversion near the ground was shown to correlate with a smooth lateral carpet transition. Flight 4 occurred in the late morning, as the ground began to warm and the temperature inversion began to disperse. Conversely, a strong temperature inversion was present during the early mornings when flight 1 took place. Figure 4b shows oscillations in PLSEL, in stark contrast to the previous example.

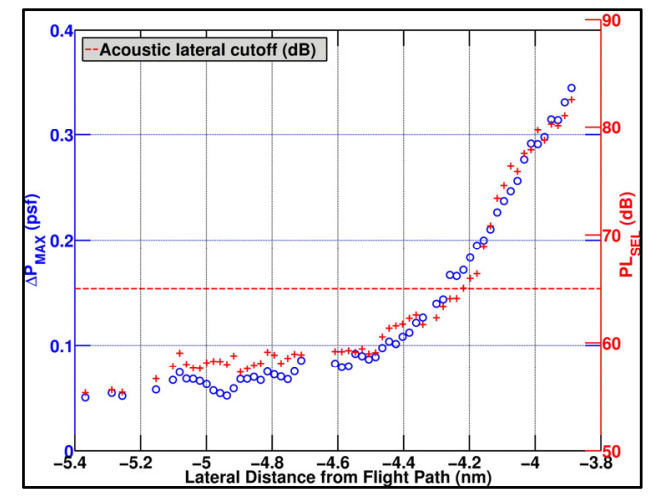

(a)

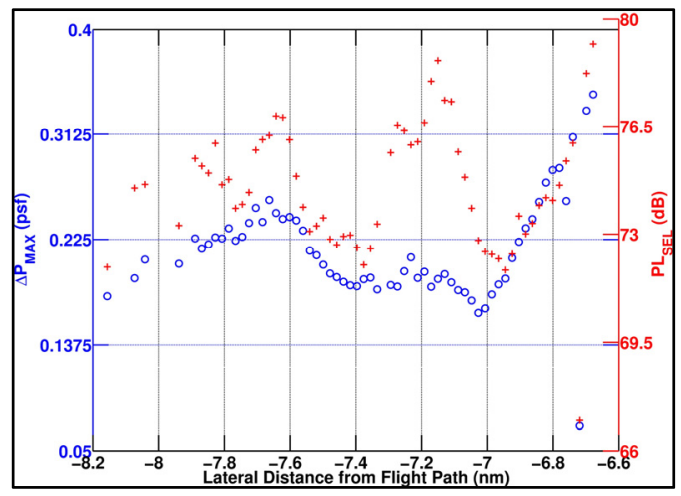

(b)

FIGURE 4. Lateral ground measurements. (a) Flight 4, pass 5; (b) Flight 1, pass 1.

An observer could infer that the lateral measurements shown in Fig. 4b are expected to quickly and exponentially decrease in magnitude from the highest level at $-6.7 \mathrm{~nm}$ to a lowest at $-8.2 \mathrm{~nm}$ under ideal conditions. The temperature inversion, however, contributes to a PLSEL of more than $76.5 \mathrm{~dB}$, nearly a nautical mile further laterally from the location of the highest measured magnitude. This can be compared to a PL $\mathrm{SEL}_{\text {below }} 60 \mathrm{~dB}$ at the same lateral distance for flight 4, pass 5 (Fig. 4a). Considering that the flight conditions for each flight pass are not substantially different $-\mathrm{a}$ difference of only -0.004 Mach number and $+6 \mathrm{~m} \mathrm{Hp}$ - it can be concluded that a strong temperature inversion can cause significant oscillations in PLSEL, greatly increasing the acoustic lateral cutoff. 


\section{Computer Model Comparison}

The major assumption of this study hinged upon the notion that modern sonic boom propagation computer models are less accurate in the transition region, and provide no solution in the shadow zone. To address this assertion, analysis was by way of comparing predicted PLSEL (PCBoom Burgers² solver) using airplane and atmospheric data as inputs, to actual sonic boom measurements near the lateral sonic boom carpet edge; an example is shown in Fig. 5. Comparisons have varying results, but each case has some common similarities that are evident in Fig. 5. First, the sonic boom carpet predicted by PCBoom is consistently narrower than the acoustic lateral cutoff because it does not model the transition zone or beyond. Microphone data consistently showed evidence of notable sonic boom levels present 1-2 nm beyond where the PCBoom solutions ended.

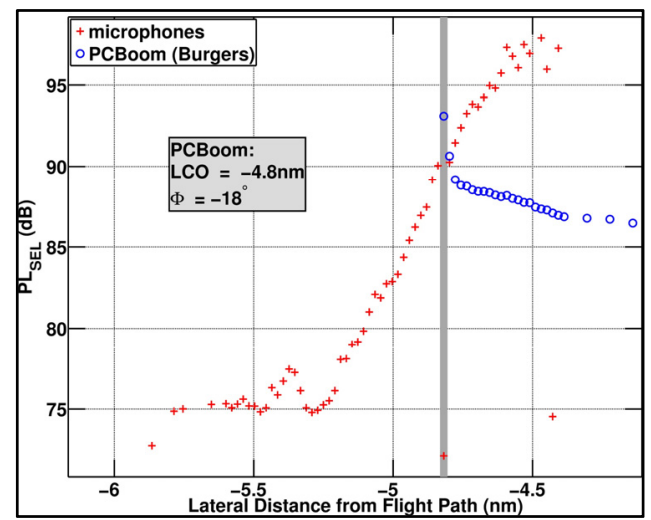

FIGURE 5. PCBoom versus measured data. Flight 2, pass 1 .

Secondly, PCBoom shows a significant $\mathrm{PL}_{\mathrm{SEL}}$ spike and complex focusing at the carpet edge, due to airplane acceleration. The spiking shows the complexity in the computer solution. Lastly, $\mathrm{PL}_{\mathrm{SEL}}$ at the edge of the PCBoom solution were higher than the microphones' for four out of the five cases.

\section{CONCLUSIONS}

Analysis of the FaINT lateral cutoff data produced several notable results. Perceived Sound Exposure Level has been suggested as a metric for further study of sonic boom signatures of the type experienced in the lateral cutoff region. Sound Exposure Level metrics have been shown to be more consistent metrics than other commonly used sonic boom metrics, such as $\mathrm{PL}_{70}, \mathrm{~L}_{\mathrm{AF}}$, and $\mathrm{L}_{\mathrm{AS}}$.

Data suggests that sonic boom PLSEL decreases in exponential fashion at the sonic boom acoustic lateral cutoff. For well-behaved lateral sonic boom profiles, PLSEL was shown to reach ambient noise at about $58.6 \mathrm{~dB}$ PLSEL. Therefore, $65 \mathrm{~dB}$ PLSEL is suggested to define the acoustic lateral cutoff of sonic booms produced during FaINT. It has been concluded that several factors affect sonic boom levels at the lateral cutoff. The most common factor during FaINT was atmospheric temperature inversions near the ground. These inversions sometimes resulted in PL $L_{\text {SEL values }}$ that were $15 \mathrm{~dB}$ higher than expected. This conclusion alone emphasizes the importance of understanding sonic boom carpet lateral cutoff phenomenon.

The definition for the physical bounds of a sonic boom carpet is well known in the industry, and is currently defined as the lateral extent that sonic boom rays are able to intersect with the ground. This paper concludes that this definition is not adequate for describing the full acoustic envelope of a sonic boom. Furthermore, more capable computer codes are required to determine the extent of sonic boom ray propagation, as common, modern computer codes do not model the considerable noise beyond lateral cutoff.

\section{REFERENCES}

1. F. Coulouvrat, "Sonic boom in the shadow zone: A geometrical theory of diffraction," Laboratoire de Modélisation en Mécanique, Université Pierre et Marie Curie, 2001.

2. J.A. Page, K.J. Plotkin, and C. Wilmer, PCBoom Version 6.6 Technical Reference and User Manual, Wyle Report WR 10-10, Wylie Laboratories Inc., Arlington, VA, 2010.

3. D.J. Maglieri, et al., "Sonic Boom: Six Decades of Research,” NASA/SP-2014-622, 2014.

4. "U.S. Standard Atmosphere, 1976." National Aeronautics and Space Administration, NASA-TM-X-74335.

5. K.P. Shepherd and B.M. Sullivan, "A Loudness Calculation Procedure Applied to Shaped Sonic Booms," NASATP-3134, 1991.

6. S.S. Stevens, "Perceived Level of Noise by Mark VII and Decibels (E)," Journal of the Acoustical Society of America, Vol. 51, Issue 2B, 1972, pp. 575-601.

7. International Organization for Standards, "Acoustics - Description, Measurement and Assessment of Environmental Noise," ISO 1996-2, 2007.

8. "American National Standard: Specification for Octave-Band and Fractional-Octave-Band Analog and Digital Filters," ANSI S1.11-2004, American National Standards Institute, Inc., 2004. 